\title{
Collaborative Authorship as Peer Mentorship
}

\section{Courtney Jacobs, Marcia McIntosh, and Kevin M. O'Sullivan}

\author{
Roses are Red; \\ Violets are Blue; \\ I want to be \\ Peer-reviewed with you. \\ -Anonymous
}

For emerging professionals and anyone unfamiliar with academic publishing, the transition from consuming to producing scholarly articles can be a daunting one. Identifying a suitable journal to publish in seems pretty manageable, but convincing yourself that you have something worthwhile to say (and we promise you do) can be more difficult. Ideally, mentorship from colleagues and peers will help you navigate this new territory. For those who do not have access to such assistance or are simply looking for additional support getting started, coauthorship can serve as a form of peer mentorship, providing the ideal environment for the uninitiated to enter the scholarly conversation. It is worth noting that the beneficiary of such support may not be someone new to the field (though it often is), as even seasoned information professionals may benefit from the experience of collaborative authorship.

Coauthorship has traditionally been more common in the sciences, where research models often necessitate team or lab-based projects and publications. Although it has not always been the norm in the humanities, collaboration has become more common in digital humanities work, where diverse skills and knowledge bases continue to drive innovation within the field. ${ }^{1}$

In an egalitarian desire to provide access to information, librarianship is, by its very nature, collaborative work. It makes sense that the profession's researching and publishing models reflect the outgrowth of these collaborations. This natural connection led us to pursue a consideration of the effectiveness of coauthorship.

During the past two years, the authors (all early-career professionals) have collaborated as a three-person team to develop the "3D Printed History of the Book Education" project, or 3Dhotbed. ${ }^{2}$ The project aims to make book history teaching tools more accessible and affordable using 3D technologies. The collaboration between our two institutions-The University of North Texas and Texas A\&M University - began in the summer of 2016. We started by scanning, modeling, and $3 \mathrm{D}$ printing hand-press period typecasting tools borrowed from Texas A\&M's Book History Workshop. The data sets for the final models were made available in the spring of 2017 and can be freely downloaded from the 3Dhotbed Collection in the UNT Digital Library. ${ }^{3}$

This experience was beneficial for us in both process and product: In less than two years of collaboration, we built a successful typecasting education toolkit, crafted a digital resource on typecasting methods, and published a scholarly article in a major 
peer-reviewed journal. ${ }^{4}$ More importantly, we forged a collaborative professional cohort that has produced significant long-term output, including innovative public humanities outreach initiatives, prominent participation in related professional service groups, career advancement, and additional publications. This editorial is an extension of this work, comprising some thoughts on the nature of successful collaborative authorship and how our experience helped us grow professionally.

\section{When One Becomes Many: Why Collaborate?}

Perhaps the greatest benefit of coauthorship is the strengthening of ideas that occurs in collaboration. Stephen Johnson argued in Where Good Ideas Come From: The Natural History of Innovation that a "majority of breakthrough ideas emerge in collaborative environments." 5

Seeking such academic partnerships within an organization or drawing from multiple disciplines can provoke more innovative work. The 3Dhotbed project exists at the crossroads of digital humanities, book history scholarship, and digital library development, encouraging not only presentations and publications in each discipline, but also the pursuit of innovation across multiple fields. Coauthorship also promotes a larger body of potential work, as the group is capable of publishing in each scholar's individual area of expertise.

Another ancillary benefit to coauthorship is the potential to decrease an individual author's time commitment to a specific research and/or publication project while increasing the team's overall productivity. Similar to the digital humanities project upon which it was based, our team's distributed work model increased our ability to research, analyze, synthesize, draft, and edit an article within our allotted time frame. Coauthorship begets further collaboration: A larger team is often more capable (and sometimes more willing) to take on additional collaborations as the nature of the original project expands to include other individuals, institutions, and projects.

Not to be overlooked is the potential for collaborative authorship to serve as a vehicle for successful peer mentorship. The notion that mentorship only exists as a hierarchical, top-down relationship is, thankfully, an outdated one. Collaborative writing encourages one to seek mentors across the field, not just across campus, and to identify and build relationships with those holding similar interests and concerns. It can also be a valuable way to build and deepen your professional network.

Among the more practical benefits is the process's potential to strengthen your skills as a writer. Peer-accountability in a group collaboration can be greatly beneficial when cultivating an effective writing practice. This is especially true for those who find it difficult to self-motivate or to turn abstractions into articles. Just as a rising tide lifts all boats, a collaborative team pools resources, essentially multiplying your community of practice from day one. Within the 3Dhotbed group, we found coauthorship to be motivating during times of difficulty. It is far easier to overcome writer's block with discussion and support from our peers than struggle with those same issues on our own.

\section{Being Intentional about Collaboration}

If a collaborative effort is to have peer mentorship benefits, it is vital that the partnership be a healthy and balanced one. As Lynne Siemens has written, "It is by (un) productive working relationships that many projects live and die. These relationships are comprised of dignity, respect, trust, and understanding among team members, at all project stages." 6

Some level of conflict is guaranteed in any group effort; the success or failure of the project in large part depends on how this is managed. It is a serious commitment to embark upon a collaborative research project. If your promotion relies upon publish- 
ing, the stakes attached to the quality and timeliness of the project's output can be very real. Therefore, it is of the utmost importance to be intentional and deliberate when establishing the collaboration.

As with any relationship, open communication and trust are paramount. We recommend having The Talk with your collaborators: "When two (or more) people love each other's ideas very much, they enter into a special relationship..." All kidding aside, it is a serious commitment to embark upon a collaborative research project. For those whose promotion relies upon publishing, the stakes attached to the quality and timeliness of the project's output can be very real. Expressing your expectations as a partner, identifying the deliverables, defining how work will be credited, and clarifying the expected level of commitment are all things a group should discuss up front. To reiterate, do not wait until submitting an article to determine who will be listed as first author, or voice your demand for an open access publication venue. Depending on the relationship among the partners, you might also consider drafting a formal agreement outlining these terms of participation and signing it together. The contract can serve as a nonpartisan authority that the group can refer to during moments of disagreement.

In transitioning from a multifaceted digital project to a collaborative authorship effort, we understood that our team dynamics would also shift. Whereas we had previously worked independently and reported back to the group, our new scholarly ambitions demanded that we work in communion. We settled upon a set of clear, attainable goals and formulated a timeline, allowing ample time to develop a rhythm of brainstorming, researching, and writing over the course of several months. In discussing projected workload, we determined each would shoulder an equal burden in writing and editing. As the project evolved, we periodically revisited the matter of author acknowledgment, agreeing on alphabetical order as the most egalitarian approach. Forgoing a formal written agreement, we met more frequently to maintain accountability to one another.

\section{Collaborative Writing: A User's Guide}

Being intentional about with whom you collaborate is just as important as determining how you collaborate. A critical question is, "How do three librarians from varying backgrounds write and edit an article using one voice?"

Numerous tools exist to assist the coauthorship process, such as Etherpad, Fidus Writer, and Slack. Since each of us was already well-versed in the Google Drive suite of tools, we decided early on to set up a shared project folder in which to store all of our materials. This struck the right balance of collaborative power, transparency, and low barrier of entry, in addition to affording us flexibility in developing our joint writing process.

We created subfolders for each new project development, such as publication drafts, full-text articles, and research-related materials. As the work expanded, these grew to include areas for presentation proposals, bimonthly meeting notes, and to-do lists. Each partner began with an open document for free-writing - an uninhibited "braindump," where we were able to think through writing and (as the saying goes) type ourselves to a good first sentence. We brought these initial ideas back to the group to see how they all fit together and developed the arc of a first draft over a conference call.

As we planned our workflow, the primary objective in the first phase was not to produce a cohesive piece, but rather to discover where our individual efforts might coalesce in the collaborative process. Many of the ideas generated in this initial phase fell by the wayside, but this falsework was not for nothing, as excised text can often form the basis for future writing projects. 


\section{Collaborative Editing: A Survival Guide}

Just as collaborative writing often produces more fruitful ideas, collaborative editing has the power to strengthen the quality of that output. Beginning with an exploratory, trust-building period during the paper's infancy contributed to a smoother editing process later on, especially when it came time to start slashing one another's prose. We also forged unspoken rules for engagement before and during our joint editing session: Come prepared, come humble, and come ready to represent your perspective.

Our method of editing consisted of an open and transparent peer-review process using suggestion mode within our Google document. This allowed each reviewer the opportunity to suggest edits without erasing their colleagues' words. Coming to the meeting prepared with notes and comments was imperative. This preliminary work allowed for deeper engagement with the material and enabled further, more impactful, progress during that session. As a group, we quickly acclimated to this practice and the expectations required for a productive meeting.

There is a level of humility required on the part of a mentee in a traditional, hierarchical mentoring relationship; we discovered this to be true within collaborative authorship as well. Initially, we were protective of our individual contributions. It soon became clear that, to create the paper we all wanted, continuing this Gollum-like possessive mentality would be untenable. As we went line-by-line through the paper, we recognized the points where we were writing divergently, discussed the issue or word choice, and reached consensus as to the best compromise. In so doing, we developed a trust in moving forward together, paragraph by paragraph. This process of collaborative editing influenced the thinking and writing style of each contributor. Over time, a "house style" emerged: Sentence length, syntax, and vocabulary choices were informed by the preferences and better practices within our peer group. Very soon, we no longer recognized the delineation among each others' words, and we moved forward drafting a single, cohesive text.

When it came time to recruit outside readers for our draft, we benefited from the wide range of expertise in each of our respective professional networks. We sent each reader a unique Google document in which they could freely edit and make comments. After a two-week turnaround, we met again as a group to digest their suggestions. As expected, feedback from the readers initiated extensive rewrites. While daunting at first, we recognized an opportunity to cut entire sections and significantly revise other portions, thus producing a paper that was far more streamlined and appropriately focused. Once satisfied, one group member reformatted the visual look and feel of the paper for consistency. We again sought advice from outside sources regarding publication etiquette and best methods for communicating with editors. We picked a primary contact and sent it off.

\section{Building an Escape Hatch: Contingency Planning}

It is important to acknowledge that not all collaborations are healthy. Conflict, though sometimes productive, can erode any possible peer-mentoring benefits if left unchecked. Jobs, personal situations, and levels of interest can all change, and disagreements may arise from issues with workload division, accountability, or failures in communication.

To mitigate the fallout of these potential risks, build an escape hatch (or a few) into your collaboration. This is especially important if you are considering a multiphase project with several products. Setting periodic deadlines with concrete deliverables can serve to ensure ongoing interest and accountability. These check-in points offer the partners opportunities to opt out in a "no fault divorce." If you find yourself in a nonbeneficial collaboration, it is preferable to have an honest discussion explaining these priority shifts as they occur than to continue in an unfavorable situation. 
Finally, it is important to note that an unproductive collaboration is not a failure. Although the group may not have met its stated goal, its members will nevertheless come away having gained valuable experience regarding the intersection of professional goals and personal relationships. Understanding how a project might have been conceptualized or implemented poorly is valuable capital that can be applied toward future endeavors.

\section{Final Thoughts}

Because of the collaborative nature of our group, the 3Dhotbed team was able to invest more deeply in the potential output of our project. From a practical standpoint, we benefited from the combined pool of available institutional resources and professional networks. But more important, our collaboration became a professionalizing experience for all involved. As we grew as scholars, we applied our findings and experiences to expand the programs and services offered at our home institutions.

Expertise and advice from your colleagues and peers can be instrumental in professional growth. Collaborative authorship is an experiential approach to mentorship, wherein peers can discern and address challenges together and share mutual successes. The practice of "thinking together" that occurs during collaborative writing inhibits isolation among new academics seeking to develop their research agenda. It is a model, in fact, that will continue to provide benefits as you progress in your role.

Peer mentorship can be a strategic avenue for professional development and an effective method for enhancing a research agenda. If you are an early-career professional interested in embarking upon a significant research project, consider seeking out like-minded peers. If you have more experience in your field, consider pulling someone else up the ladder along with you. Doing so will not only increase the overall quality of your project; it will also improve the individual academic advancement of each team member.

\section{Courtney Jacobs Head of Outreach and Community Engagement for UCLA Libraries Special Collections} Marcia McIntosh Digital Production Librarian at the University of North Texas Libraries

Kevin M. O'Sullivan Curator of Rare Books \& Manuscripts at Texas A\&M University Libraries

\section{Notes}

1. Lisa Spiro, "Collaborative Authorship in the Digital Humanities," Digital Scholarship in the Humanities (blog), April 29, 2008, available online at https://digitalscholarship.wordpress. com/2009/04/21/collaborative-authorship-in-the-humanities/ [accessed 3 May 2018].

2. More information is available at http://3dhotbed.info [accessed 26 April 2018].

3 . The full collection of tools and statistics regarding their usage are available at https://digital.library.unt.edu/explore/collections/THREED/ [accessed 26 April 2018].

4. Courtney Jacobs, Marcia McIntosh, Kevin M. O'Sullivan, "Making Book History: Engaging Maker Culture and 3D Technologies to Extend Bibliographical Pedagogy," RBM 19, no. 1 (Spring 2018): 59-69

5. Steven Johnson, Where Good Ideas Come From: The Natural History of Innovation (New York: Riverhead Books, 2010), 228.

6. Lynne Siemens, "'It's a Team if You Use "Reply All”': An Exploration of Research Teams in Digital Humanities Environments," Literary and Linguistic Computing 24, no. 2 (June 2009): 228. 